Check for updates

Cite this: J. Mater. Chem. A, 2022, 10 , 2122

Received 29th October 2021

Accepted 2nd January 2022

DOI: 10.1039/d1ta09286c

rsc.li/materials-a

\title{
Interface effects from moisture in nanocomposites of 2D graphene oxide in cellulose nanofiber (CNF) matrix - A molecular dynamics study $\dagger$
}

\author{
Hanieh Mianehrow, (D) Lars A. Berglund (D) and Jakob Wohlert (D)*
}

\begin{abstract}
Molecular dynamics (MD) simulation is used to study effects from moisture at cellulose nanofibril (CNF)graphene oxide (GO) interfaces adhering in the wet state. Two CNF models with different surface chemistry are used: native cellulose nanofibrils (NCNF) and TEMPO-oxidized cellulose nanofibrils (TOCNF). Work of adhesion and interfacial shear strength at the CNF-GO interface is compared with $\mathrm{CNF}$-graphene interfaces to interpret interaction mechanisms. Any trapped interfacial water reduces adhesion strongly. Results show, however, that there is a meta-stable state where water is initially present at the CNF-GO interface but disappears with time to form a dry interface. Once a solventexcluded interface is formed between CNF and GO, the surface oxidation has little effect on molecular adhesion. We propose that the main adhesion mechanism is entropy gain from release of water molecules as CNF and GO jump into contact when brought close together. Hydrogen bonding does not govern the work of adhesion between CNF and GO, but plays an important role for interfacial shear strength.
\end{abstract}

\section{Introduction}

Polymers are strongly reinforced by well-dispersed 2D nanoplatelets oriented in-plane. Young's modulus and tensile strength can reach high values for such nanocomposites, especially for strong, high aspect ratio nanoplatelets. ${ }^{1}$ Graphene, ${ }^{2,3}$ graphene oxide, ${ }^{4}$ clay, ${ }^{5}$ molybdenum disulfide ${ }^{6}$ and boron nitride ${ }^{7}$ are examples of 2D reinforcements which, besides mechanical properties, also can provide good gas barrier properties, fire retardancy, electrical properties and thermal conductivity.

Many nanoplatelets have a thickness of only approx. $1 \mathrm{~nm}$, so the specific surface area and the cross-sectional interface density (number of interfaces per unit thickness) in nanocomposites can be very high. The nanoplatelet/matrix interfacial interactions are thus critical during formation of the nanostructure, but they also offer tremendous opportunities for unique nanocomposite properties if molecular adhesion mechanisms ${ }^{8}$ can be controlled. Here, we are using molecular dynamics (MD) to simulate interfacial interactions (work of adhesion, interfacial shear strength) in cellulose-graphene oxide and graphene nanocomposites and investigate mechanisms for moisture effects. Results can guide processing efforts

Department of Fibre and Polymer Technology, Wallenberg Wood Science Center, KTH Royal Institute of Technology, Teknikringen 56, 10044 Stockholm, Sweden. E-mail: jacke@kth.se

† Electronic supplementary information (ESI) available. See DOI: 10.1039/d1ta09286c for high volume fraction nanocomposites ${ }^{9}$ and may inspire new drying procedures but also ideas for chemical surface modification of the nanoparticles. The interface is critical for the strength/toughness balance in $2 \mathrm{D}$ nanocomposites, ${ }^{\mathbf{1 0}}$ and simply needs to be better understood.

Cellulose nanofibrils $(\mathrm{CNF})^{5,11}$ constitute the matrix phase in the present nanocomposite models. Experimentally, after vacuum filtration and drying, CNF hydrocolloids can form almost non-porous high-strength films with flexible CNF fibrils (approx. $4 \mathrm{~nm}$ by $1 \mu \mathrm{m}$ ) in swirled, densely packed structures. ${ }^{12}$ Most likely, large capillary forces ${ }^{\mathbf{1 3}}$ are responsible for film formation during drying, and fibrils are plastically deformed ${ }^{\mathbf{1 4}}$ so that nanoscale porosity is reduced. The first $2 \mathrm{D}$ nanoplatelet composites with CNF matrix were prepared in our lab ${ }^{15,16}$ simply by mixing two hydrocolloids (one with CNF and one with nanoplatelets) followed by vacuum filtration. CNF offers considerable advantages in the development of more sustainable nanomaterials. In addition, polymer matrix nanocomposites based only on physically interacting nano-objects can be readily functionalized and possibly recycled.

Graphene oxide, the oxidized derivative of graphene, can have a Young's modulus of around $250 \mathrm{GPa} .{ }^{17}$ The oxidation introduces different functionalities such as hydroxyl, epoxy and carboxyl groups, ${ }^{18,19}$ which facilitates GO dispersion in water and reduces the aggregation problems observed for graphene.

The properties of both CNF-based ${ }^{20-23}$ and GO-based ${ }^{24-26}$ nanomaterials are influenced by moisture. Barthelat and colleagues showed that GO membranes can be given complex shapes in the wet state by the use of $25 \%$ of cellulosic slurries of 
fibres $50-2600 \mu \mathrm{m}$ in length. ${ }^{27} 3 \mathrm{D}$ printing of CNF inks is a real possibility, ${ }^{28}$ where it should be possible to include $2 \mathrm{D}$ nanosheets. For CNF-GO nanocomposites, there are not so many previous studies, ${ }^{29-32}$ whereas graphene or reduced GO have been used more frequently ${ }^{30,33-36}$ when high electrical conductivity is desirable. (A table comparing mechanical properties reported in the literature is presented in ref. 29). We have recently investigated the mechanical behaviour of CNF-GO nanocomposites, ${ }^{29}$ and carefully characterized the nanostructure using wide angle $\mathrm{X}$ ray scattering (WAXS) and small angle X-ray scattering (SAXS) techniques. The gas barrier properties were influenced by moisturizing-drying cycles, where interfacial interactions seemed to improve by moisture cycling, although in general, aspects of molecular adhesion effects are poorly understood.

There are some interesting previous molecular scale investigations of the cellulose-GO interface. As a background, one may note that moisture at cellulose-cellulose interfaces increases ductility in films from nanocellulose rods $^{37}$ and that also GO-GO interaction is influenced by moisture. ${ }^{38}$ The cellulose-GO interfaces are, however, specific and need careful investigation. Rahman et $a l .{ }^{39}$ investigated a model graphene-amorphous cellulose composite and identified basic parameters of importance for mechanical behaviour. Mao et ll $^{\mathbf{4 0}}$ simulated XRD spectra in GOcellulose nanocrystal composites, and interpreted mechanisms for changes in the (200) lattice parameters from interactions with GO. Alqus et $a l .{ }^{41}$ performed MD simulations of native cellulose and graphene in water. They found a stable, "solvent-excluded" (no water) interface between graphene and the "hydrophobic" cellulose face in water. Zhu et $a .^{42}$ investigated GO-TEMPO (2,2,6,6-tetramethylpiperidine-1-oxyl radical)-mediated oxidized CNF systems with small GO sheets and large CNF "fibres", with focus on the material system rather than on interfacial adhesion details. The most relevant previous study to the present work was by Tsukruk and colleagues, ${ }^{43}$ who combined experiments and MD simulations to investigate the assembly of GO and oxidized TEMPO-CNF in water. Focus was on effects from GO oxidation degree and formation of the nanoobjects, although the role of water was not analysed in detail. Simulations showed the importance of, in particular, the van der Waals interactions across the interface.

Here, molecular adhesion is investigated at $\mathrm{CNF} / \mathrm{GO}$, TEMPO-CNF/GO and graphene interfaces in the presence of water. The objectives are to quantify work of adhesion and interfacial shear strength, describe interaction mechanisms and clarify the role of water for materials design purposes. In the lowest energy state (equilibrium), there is no water present at the interfaces, but if water becomes kinetically trapped (e.g., during drying) it substantially reduces adhesion. In either case, the net effect from hydrogen bonding is negligible. The potential of interface tailoring of nanocomposites at molecular scale is apparent from the present results.

\section{Methods}

\section{Model development and molecular dynamics simulations}

A graphene model was constructed from 1472 carbon atoms in a hexagonal lattice with lateral size $7 \mathrm{~nm} \times 6 \mathrm{~nm}$. Carbon atoms on the edges of the sheet were covalently bonded to the opposite side of the sheet over the periodic box boundary, which makes the graphene sheet infinitely large. The graphene was further randomly functionalized on both sides by adding hydroxyl and epoxy groups to make GO. The total epoxy to hydroxyl ratio was $25 \%$ to resemble common GO made by Hummer's method. ${ }^{\mathbf{4 4}}$

The native cellulose nanofibril (NCNF) model was constructed from 18 glucan chains, each with 8 glucose units, arranged in a hexagonal cross-section, which currently is the most accepted model $^{45-48}$ (Fig. 1c and d). This NCNF model is a result of cellulose biosynthesis arguments, ${ }^{49,50}$ with support from SAXS data. ${ }^{51}$. In the TEMPO-oxidized cellulose nanofibril (TOCNF), every other glucose unit in the surface chains was substituted for a glucuronic acid unit to represent highly oxidized TOCNF. Carboxyl groups were arranged such that they were all exposed to the outside of the crystal. Chains in the centre of the TOCNF crystal remained chemically identical to NCNF (Fig. 1e and f).

Two "nanocomposite" systems of NCNF-GO and TOCNF-GO were built by placing the CNFs in close proximity of the GO, with the crystallographic ( $\left.\begin{array}{lll}2 & 0 & 0\end{array}\right)$ plane of the CNFs exposed to the GO (Fig. 19). Simulations in which the (1 110$)$ plane was exposed to the GO were also performed. However, during equilibration the CNF spontaneously rotated to expose its $\left(\begin{array}{lll}2 & 0 & 0\end{array}\right)$ plane to the GO, indicating that this is the most stable configuration in line with what was reported by Alqus et al. ${ }^{41}$ Finally, $\mathrm{Na}^{+}$counter ions were added to the TOCNF-GO system to neutralize the negative charge. Systems with graphene were built in a similar manner.

MD simulations were performed using GROMACS 2019.4 (ref. 52) with a basic time step of 1 fs. The non-bonded interactions used a cut-off distance of $1.2 \mathrm{~nm}$ and long-range electrostatic interactions were employed using PME. ${ }^{53,54}$ Bonds were kept rigid at their equilibrium lengths using P-LINCS. ${ }^{55}$ The pressure was maintained at $1 \mathrm{~atm}$ using Parrinello-Rahman barostat ${ }^{56}$ with semi-isotropic coupling and temperature was maintained at 298 K using a Nosé-Hoover thermostat. ${ }^{57,58}$ GLYCAM06 (ref. 59) parameters were used for the carbohydrates, combined with $\mathrm{GAFF}^{60}$ for graphene/GO, and the TIP3P ${ }^{61}$ water model. Parameters for the $\mathrm{Na}^{+}$ions were taken from ref. 62 .

Since the purpose of the study is to investigate the effect of moisture at the CNF-GO interface, MD simulations of the CNFGO systems were performed both in a box filled with water, and in vacuum. For the CNF-GO nanocomposites immersed in water, the CNF and GO were positioned close to each other, see Fig. 1g, but each particle was completely surrounded by liquid water. Each system was equilibrated for $10 \mathrm{~ns}$. To study the effect of drying history, the dry nanocomposites in vacuum were equilibrated, wetted in water and equilibrated for an additional period of 10 ns. This condition is termed "dry-wetted". The VMD package was used to visualize simulated nanocomposite systems. ${ }^{63}$ All systems were energy-minimized using steepest descents prior to simulations.

\section{Potential of mean force calculations for work of adhesion}

The potential of mean force (PMF) between the CNF and the GO in different environments was calculated to investigate molecular adhesion and estimate work of adhesion $W_{\text {A. }}$ Umbrella 
a.

b.

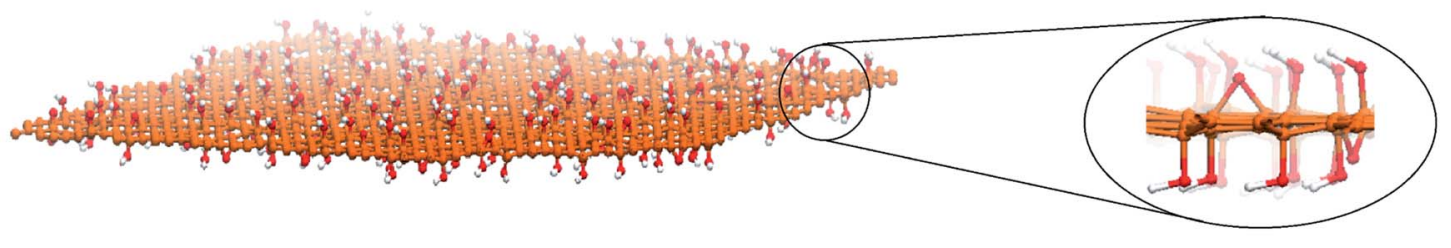

c.

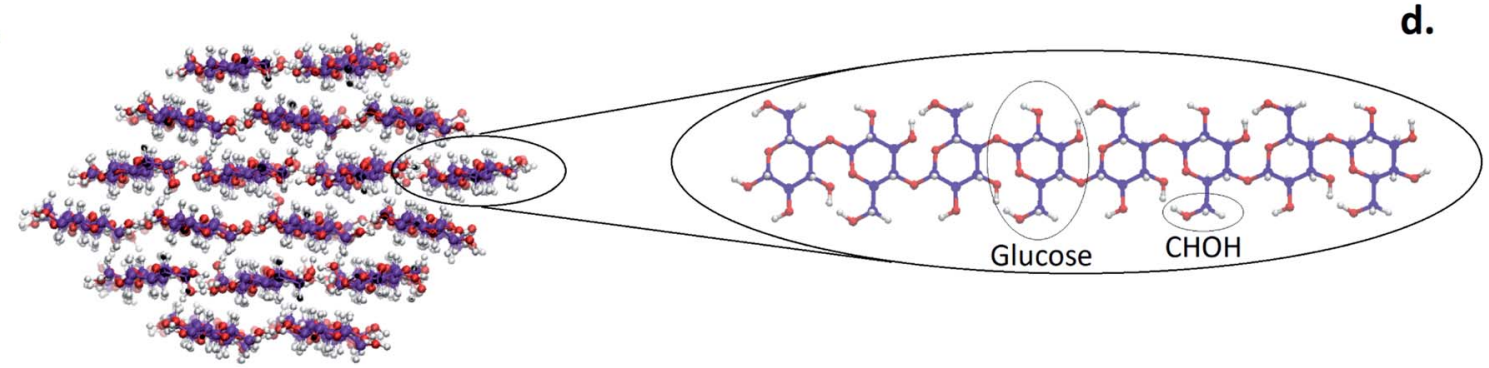

e.

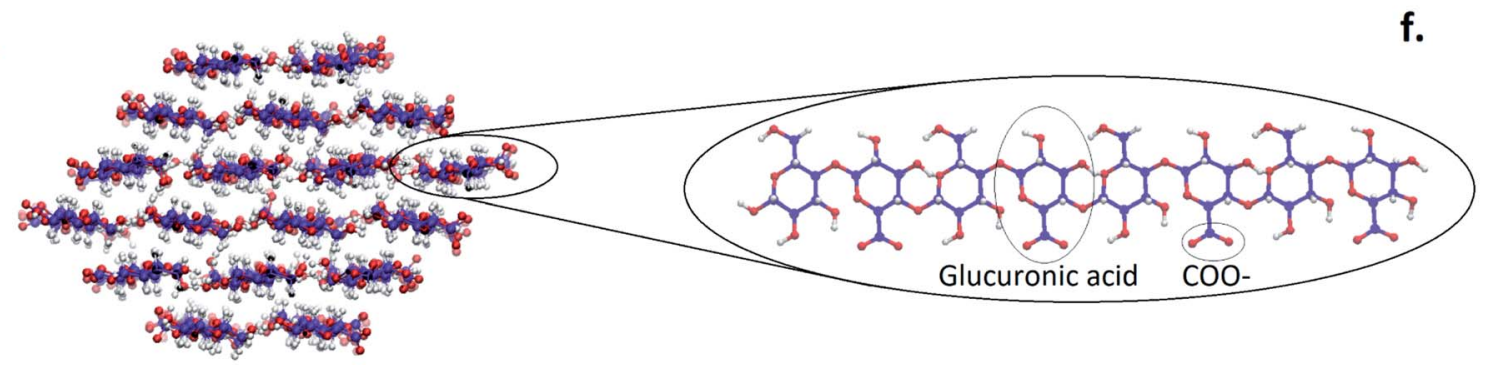

g.

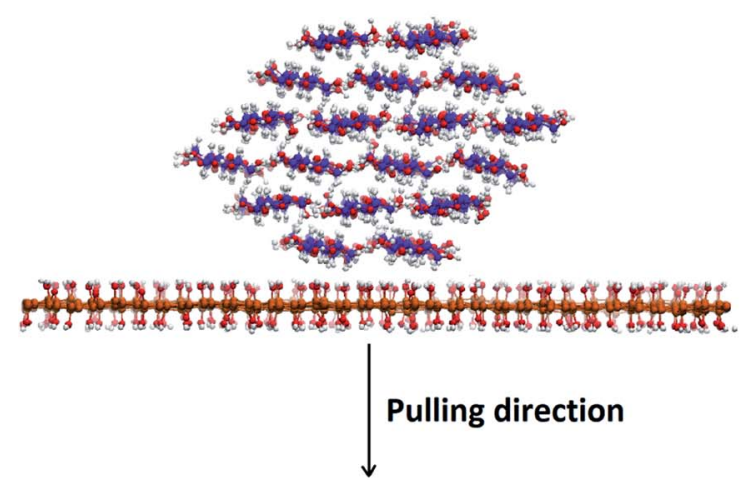

Shear direction

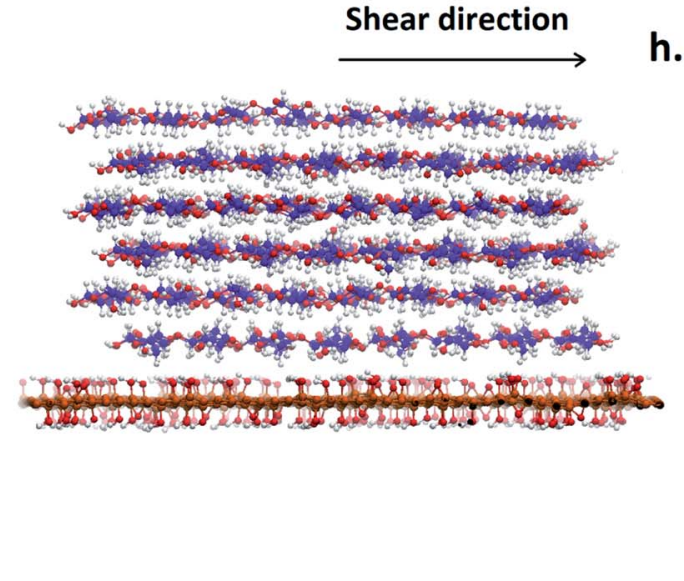

h.

Fig. 1 Infinite Graphene Oxide (GO) sheet model $(7 \mathrm{~nm} \times 6 \mathrm{~nm}$ ) with 336 carbon rings (a) with hydroxyl and epoxy groups on its surface (epoxy/ hydroxyl $=0.25$ ) (b). Finite native cellulose crystal (NCNF) model consisting of 18 chains, each with 8 glucose unites ( $5 \mathrm{~nm}$ in length) (c). Chemical structure of one native cellulose chain is shown in (d). Finite TEMPO-oxidized cellulose crystal (TOCNF) model, in which internal chains are intact but surface chains are oxidized; every other glucose unit is substituted with glucuronic acid unit. (e). Chemical structure of one oxidized chain is shown in ( $f$ ). Cross-section of CNF-GO system and pulling direction for potential of mean force and work of adhesion $\left(W_{\mathrm{A}}\right)$ calculations $(\mathrm{g})$ and side view (perpendicular to cross-section) of CNF-GO system and during interfacial shear loading of the interface to estimate interfacial shear strength (h).

sampling was used, where the umbrella simulations used the centre-of-mass distance between the $\mathrm{CNF}$ and the GO as reaction coordinate. 101 intermediate states in $0.5 \AA$ intervals were used for umbrella simulations in the CNF-GO systems. These states were prepared by starting from each equilibrated structure and then pull the CNF-GO complex apart by varying the reference centre-of-mass distance continuously during $50 \mathrm{~ns}$ of MD. Here, positional restraints were applied on the CNF and 
a spring constant of $10000 \mathrm{~kJ} \mathrm{~mol}^{-1} \mathrm{~nm}^{-1}$ was used. For the umbrella sampling, each state was simulated for $1 \mathrm{~ns}$ and a harmonic restraining potential acting on the centre-of-mass distance between the CNF and the GO was employed with a force constant of $50000 \mathrm{~kJ} \mathrm{~mol}^{-1} \mathrm{~nm}^{-1}$. No additional positional restraints were applied during the umbrella sampling simulations. Weighted histogram analysis (WHAM) ${ }^{64}$ was used to construct the full PMF for each system. This gives the free energy of separation between the CNF and the GO and can be converted into work of adhesion by normalizing the results over the initial contact area. The contact area was calculated based on the projected area of the $\mathrm{CNF}$ on the GO/graphene (see Fig. S1†), which may be conservative, since the real area of molecular contact may be smaller than this. The error bar for each point in PMF was estimated using bootstrapping. ${ }^{65}$

\section{Interfacial shear strength calculations for shear slippage between CNF and GO}

Interfacial shear strength between reinforcement and surrounding matrix is a key property for composites and is critical for reinforcement pull-out mechanisms during failure, which influence tensile strength and fracture toughness. ${ }^{\mathbf{6}}$ Shear slippage between the GO or graphene platelet and the CNF matrix was induced using constant force loading. The force was applied to the CNF centre-of-mass in the longitudinal CNF direction (Fig. 1h), so that shear is induced at the interface. Depending on the system, the force was varied in steps of 10$100 \mathrm{~kJ} \mathrm{~mol}^{-1} \mathrm{~nm}^{-1}$ until slippage occurred within a $10 \mathrm{~ns}$ simulation. Positional restraints were applied on the $\mathrm{GO} /$ graphene. Interfacial shear strength was determined as the minimum force required to slide the CNF on the GO or graphene, divided by the initial contact area.

\section{Results and discussion}

CNF and GO interface interactions were investigated in different environments to support interpretation of moisture effects on global mechanical behaviour of $\mathrm{CNF} / \mathrm{GO}$ nanocomposites. Native NCNF-GO and oxidized TOCNF-GO were simulated immersed in water, in the dry vacuum state and also in dry-wetted state where the dry nanocomposites in vacuum were subsequently exposed to water (see Methods).

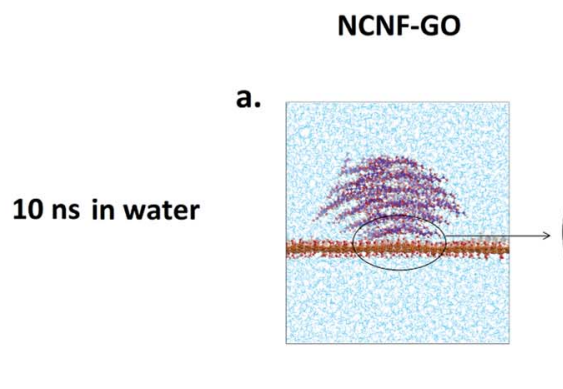

c.

$100 \mathrm{~ns}$ in water
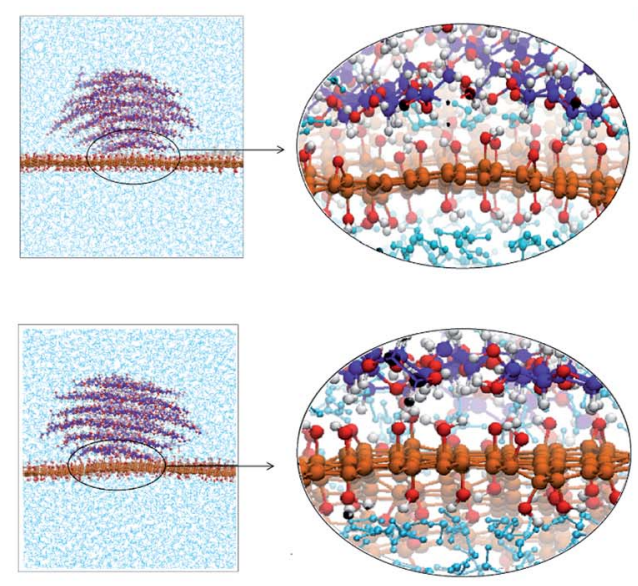

e.
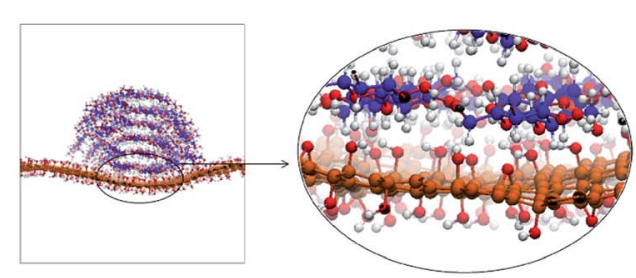

f.

$10 \mathrm{~ns}$ in vacuum

(2)

Vacuum + $10 \mathrm{~ns}$ in water b.
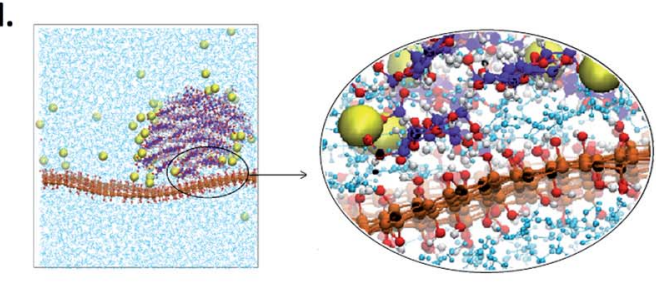

TOCNF-GO

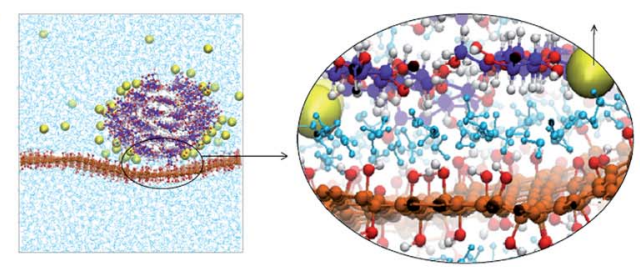

d.

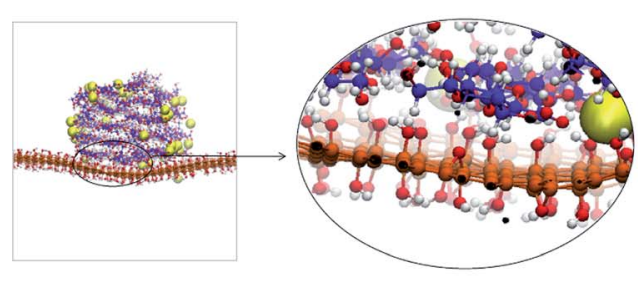

h.

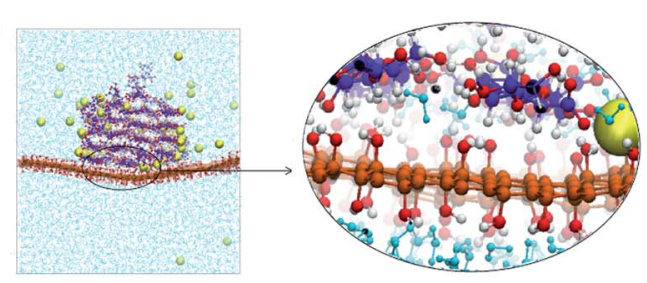

g.

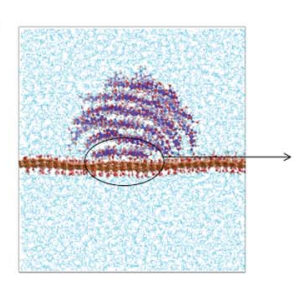

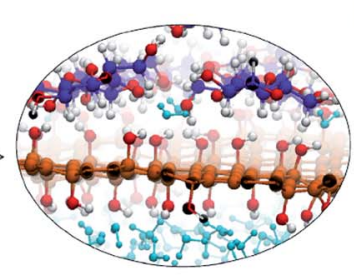

Fig. 2 NCNF-GO and TOCNF-GO systems after 10 ns adsorption time in water ( $a$ and b), 100 ns adsorption time in water (c and d). (e and f) Show results for the case of no water, after $10 \mathrm{~ns}$ in dry vacuum state. (g and h) Show results after 10 ns dry in vacuum +10 ns in water (dry + wetted). Zoomed-in interfaces are shown separately for each case. 


\section{The CNF-GO interface}

Fig. 2a and b show the final configurations after 10 ns unconstrained simulation in water. Spontaneous adsorption of both types of CNF to the GO is observed, where they jumped into contact. In both cases, however, water is present at the CNF-GO interface. Note that there is more water at the TOCNF-GO interface due to the $\mathrm{Na}^{+}$ions surrounding TOCNF in water. Zonghui et $a .^{67}$ reported that the presence of monovalent counter-ions physically increases the distance between two adjacent cellulose crystals and hence decreases the molecular adhesion between them. Here we observe a similar effect from $\mathrm{Na}^{+}$counter-ions in TOCNF-GO system, but the question is whether the water layer at the interface would disappear after longer simulation times?

Fig. $2 \mathrm{c}$ and $\mathrm{d}$ show the configurations after $100 \mathrm{~ns}$ simulation in water. Now the water layer has disappeared for NCNF-GO, while it remains at the TOCNF-GO interface even after $100 \mathrm{~ns}$. In Fig. 2e and f, both systems are simulated in vacuum for $10 \mathrm{~ns}$, with no water present. The CNF quickly adheres to the GO, and since there is no water, the CNF and GO can immediately form a tight interface with molecular/atomic contact (Fig. 2e and f). Note from the images that the CNF deforms strongly as it adheres to GO due to the strong adhesion forces. Finally, the dry nanocomposites in vacuum were wetted (Fig. $2 g$ and $h$ ). These dry-wetted nanocomposites largely retain the tight interfaces formed in the dry state, even after longer (100 ns) times, and only a few water molecules are observed at the interface between the CNF and the GO.

These simulations show that water may become kinetically trapped at the interface between CNF and GO. This is expected to influence mechanical properties. For native NCNF immersed in water, water leaves spontaneously, forming a dry NCNF/GO interface. This case of "solvent-excluded contact" then represents the global equilibrium and the kinetically trapped water condition is temporary. For TOCNF however, simulation results are not conclusive about which state is lower in energy: with or without water at the interface. Therefore, the work of adhesion between CNF and GO is further investigated in the following a.

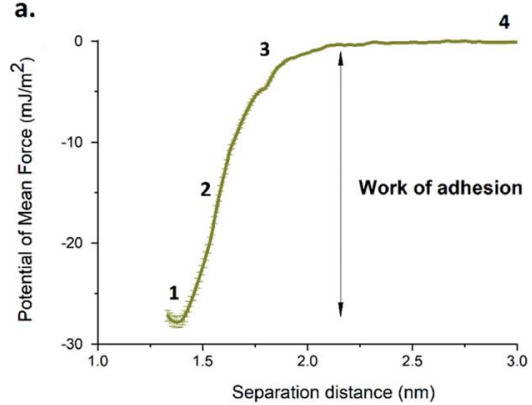

b.

1

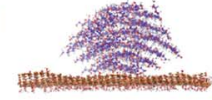

3

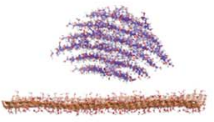

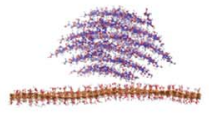

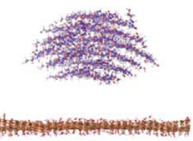

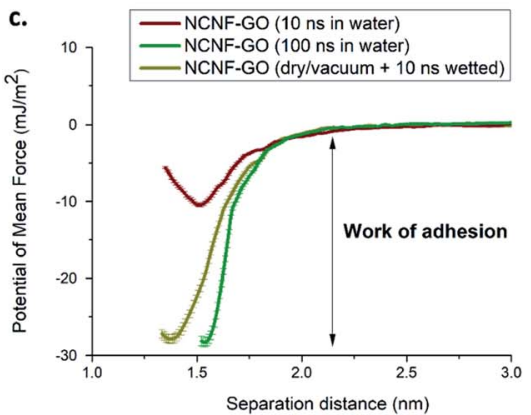

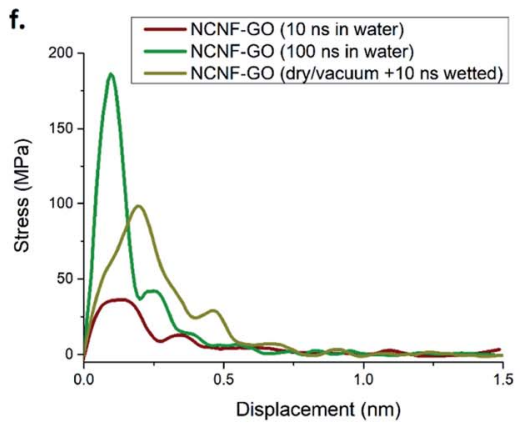

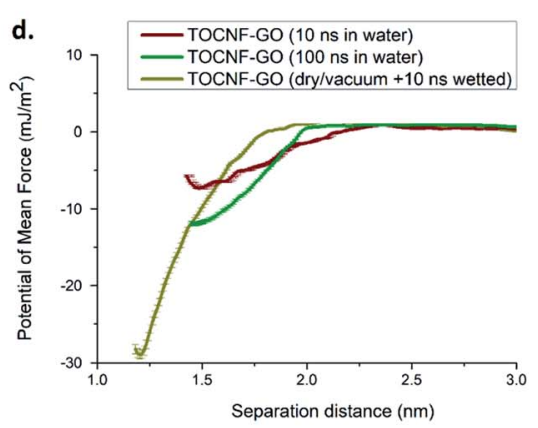

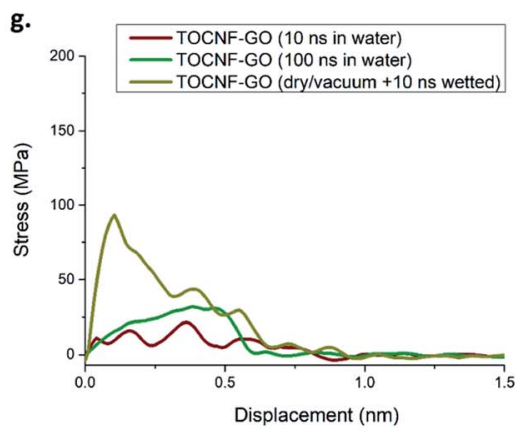

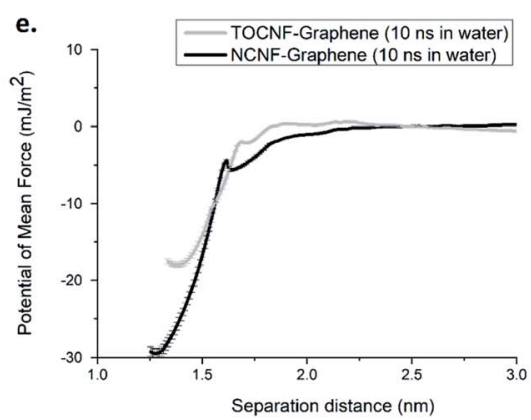

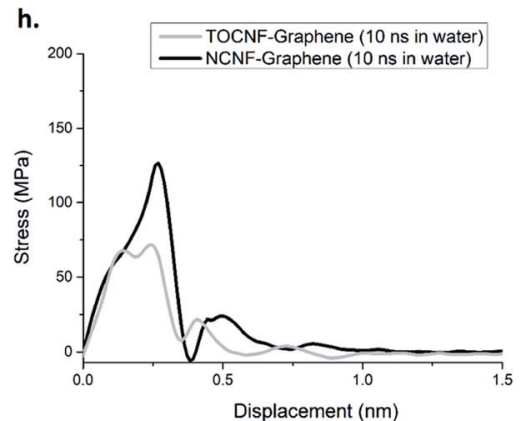

Fig. 3 An example of potential of mean force (PMF) graph normalized by contact area for NCNF-GO (a) and corresponding trajectories at different stages of separation (b), PMF graphs for NCNF-GO (c), TOCNF-GO (d) and NCNF/TOCNF-graphene (e) in different states. Stress versus displacement graphs for NCNF-GO (f), TOCNF-GO (g) and NCNF/TOCNF-graphene (h) in different states. 
section. As expected, there is no water inside the CNFs and moisture effects are dominated by interparticle interface effects.

\section{Interfacial adhesion}

Fig. 3 shows potential of mean force (PMF) versus separation distance between CNF and GO, measured perpendicular to the interface. The reference level is selected as the fully separated state, where there is no interaction between the CNF and GO, since this state is the same across the different simulations. Here, the value of the PMF is zero. In this representation, the PMF is the free energy per unit contact area gained by moving the CNF and GO to a separation distance $z$, relative to the noninteracting state. The work of adhesion, $W_{\mathrm{A}}$ thus is the lowest PMF value, which is obtained at contact but with the opposite sign (Fig. 3a). The use of umbrella sampling presupposes (local) equilibrium conditions. This facilitates the thermodynamic analysis and is actually necessary to estimate $W_{\mathrm{A}}$, since it is an equilibrium property by definition. CNF-GO trajectories at different separation distances are shown in Fig. 3b.

Fig. 3 also shows the effect of water at the CNF-GO interface. The $W_{\mathrm{A}}$ for NCNF-GO after $10 \mathrm{~ns}$ in water is much lower than for NCNF-GO after $100 \mathrm{~ns}$ in water (Fig. 3c). After $10 \mathrm{~ns}$, there is still water at the interface, but this has disappeared after $100 \mathrm{~ns}$. Interestingly, the dry-wetted interface gives similar $W_{\mathrm{A}}$ as for the 100 ns NCNF-GO case for simulation in water. This supports the previous observation that water does not penetrate into the interface during soaking of a dry interface in water. We can now draw conclusions about water in the TOCNF-GO nanocomposite interfaces: a similar $W_{\mathrm{A}}$ trend is observed for TOCNF-GO in Fig. $3 \mathrm{~d}$ as for the previous NCNF-GO. The $W_{\mathrm{A}}$ is higher for TOCNF-GO after $100 \mathrm{~ns}$ in water than after $10 \mathrm{~ns}$ in water, since water is leaving the interface after a longer equilibration time, compare Fig. $2 \mathrm{~b}$ and d. The dry-wetted interface even gives the highest $W_{\mathrm{A}}$ in this case. The reason is that for TOCNF-GO, some water remains at the interface even after 100 ns (Fig. 2d). $W_{\mathrm{A}}$ data for each system is reported in Table 1. A similar behaviour was recently shown in MD simulations of interacting NCNFs in water. ${ }^{68}$

The difference between the $W_{\mathrm{A}}$ of dry-wetted TOCNF-GO and TOCNF-GO after $100 \mathrm{~ns}$ in water, suggests an influence of the drying history. It indicates that the hydrated interface is a meta-stable state and that the hydration/dehydration process is kinetically controlled and the stable state is essentially a dry TOCNF-GO interface. In essence, this is analogous to the concept of cellulose fibril co-crystallization (hornification), which is the term used for the strong aggregation of native cellulose fibrils when dried from wet state. ${ }^{69-71}$

In an attempt to quantify interfacial interactions in dry/ vacuum state, we observed very high adhesion forces between CNF and GO. For this case, the CNF does not detach completely from GO during the separation simulations and the PMF graph does not level off (Fig. S3†). The interfacial adhesion forces are so strong that if no positional constraints are used for CNF, the CNF itself is failing during separation simulations. This is visualized in Fig. $\mathrm{S} 4 \dagger$ and the $W_{\mathrm{A}}$ in dry/vacuum state is therefore reported as $\geq 120$ in Table 1 .

To investigate the significance of GO surface oxidation for CNF-GO, simulations of NCNF-graphene and TOCNF-graphene were performed in water and the corresponding $W_{\mathrm{A}}$ were calculated. Fig. 3e compares the PMF curves for NCNF-graphene and TOCNF-graphene. Here, $10 \mathrm{~ns}$ of simulation was enough to achieve a completely solvent-excluded, dry interface between the CNF and the graphene (no interfacial water, Fig. S2 $\dagger$ ). Interestingly, the meta-stable state (hydrated interface) is observed as a small dent at around $1.7 \mathrm{~nm}$ separation, which corresponds to one hydration layer between CNF and graphene. For the case of graphene, this state is relatively shortlived. Both NCNF-graphene and TOCNF-graphene exhibit similar behaviour, although TOCNF-graphene has lower $W_{\mathrm{A}}$, again possibly due to the presence of $\mathrm{Na}^{+}$counter ions.

In the Fig. $3 \mathrm{f}-\mathrm{h}$, the same data is replotted as stress $v s$. displacement for pedagogical purpose. The slope of the PMF curve is simply translated into "stress", although this is most often a macroscopic parameter. The specific procedure (see Methods) may influence the shape of the curve and is introducing uncertainties in this respect. The area under the stressdisplacement curve is, however, of high accuracy. Here, the displacement is measured from the point where the stress changes sign, which is the equilibrium separation distance. The maximum value in these curves is the interfacial adhesion strength. This data is also reported in Table 1 for each case. Note that these stress levels for molecular adhesion are

Table 1 Number of hydrogen bonds between CNF and GO, Potential Mean Force (PMF), work of adhesion and longitudinal interfacial shear strength for different systems at different hydration states. The data are reported for $10 \mathrm{~ns}$ equilibrated interfaces

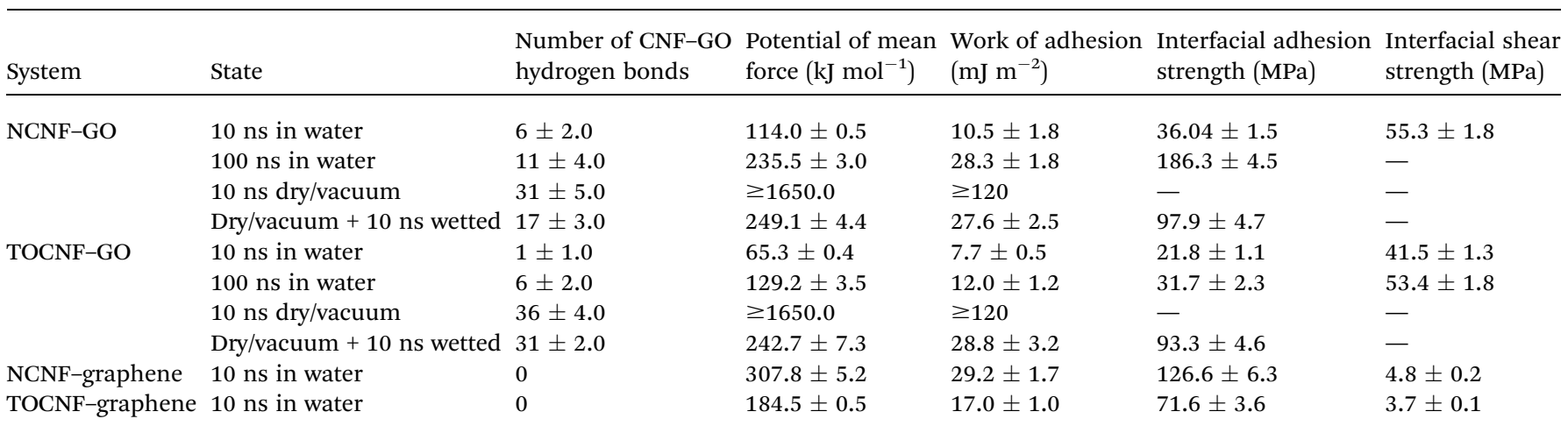


expected to be higher than stresses measured in micromechanical experiments involving cellulosic fibres, due to factors such as contaminants or non-ideal molecular scale contact. Fig. 3f shows that for NCNF-GO the strength is the lowest when there is water present at the interface. Once the interface becomes solvent-excluded (dry) the interfacial strength increases drastically. It is interesting that the strength is much higher for 100 ns adsorption in water than the drywetted structure, although $W_{\mathrm{A}}$ is the same for both. The reason for similar $W_{\mathrm{A}}$ is that lower strength interfaces carry higher stress at larger displacements. A similar trend for moisture effects is observed for TOCNF-GO where the wet interfaces both show lower adhesion strength than the solvent-excluded interface (Fig. 3g). This is consistent with results from a MD study on acetylated CNF which shows that the lack of solvent-excluded interface between acetylated CNFs reduces $W_{\mathrm{A}}$ significantly. ${ }^{72}$ Importantly, with no water at the interface, both NCNF-GO and TOCNF-GO exhibit similar work of adhesion (Table 1). In the case of graphene, the strength is higher for NCNF-graphene than TOCNF-graphene (Fig. 3h), possibly due to the presence of counter ions in the latter case.

A general observation from Fig. $3 \mathrm{c}-\mathrm{h}$ is that water is apparently the main factor influencing curves of PMF versus separation distance and $W_{\mathrm{A}}$, despite the substantial interface differences between these four systems, (NCNF-GO, TOCNFGO, NCNF-graphene, TOCNF-graphene). When there is no water at the interface, the $W_{\mathrm{A}}$ is similar in all cases, and for NCNF-GO, TOCNF-GO and NCNF-graphene, they are the same. Thus, in the following sections, various interaction mechanisms are analysed.

\section{Interaction mechanisms for work of adhesion}

To analyse the adhesion, it is important to study the work of adhesion between CNF and GO as calculated in the previous section. It is defined by the difference in total excess Helmholtz' free energy, $\Delta A^{\mathrm{ex}}$, between the adsorbed and desorbed states. Since $\Delta A^{\text {ex }}$ is an excess property, it is independent of concentration effects from CNF or GO. Using the familiar relation $\Delta A=$ $\Delta E-T \Delta S$ (where the superscript is dropped for convenience) it is possible to analyse energetic $(\Delta E)$ and entropic $(\Delta S)$ contributions to $W_{\mathrm{A}}$ separately, and also to further decompose these parts into contributions from specific interactions.

The typical explanation for interfacial adhesion in cellulose materials is strong ability of cellulose surfaces to form hydrogen bonds. It has indeed been suggested that hydrogen bonds between CNF and GO are responsible for interfacial adhesion. ${ }^{\mathbf{4 0}}$ Considering that a typical bond energy for a hydrogen bond is $20 \mathrm{~kJ} \mathrm{~mol}^{-1},{ }^{73}$ the $W_{\mathrm{A}}$ 's obtained here for the dry-wetted systems correspond to breaking one hydrogen bond per $\mathrm{nm}^{2}$, which is quite reasonable. The summary in Table 1 shows correlation between hydrogen bonds and work of adhesion, which seems enough to conclude a strong effect from hydrogen bonds. However, this conclusion is actually wrong. First of all, interfacial hydrogen bonds in wet systems can be readily replaced by hydrogen bonds to water molecules, e.g., when CNF and GO are separated. Indeed, the total number of hydrogen bonds in the
Table 2 The number of hydrogen bonds, including water, for CNF$\mathrm{GO}$ and CNF-graphene systems in water before and after separation

\begin{tabular}{lll}
\hline System & $\begin{array}{l}\text { Total H-bonds before } \\
\text { separation }\end{array}$ & $\begin{array}{l}\text { Total H-bonds after } \\
\text { separation }\end{array}$ \\
\hline NCNF-GO & $17954 \pm 13$ & $17964 \pm 7$ \\
TOCNF-GO & $18458 \pm 8$ & $18515 \pm 10$ \\
NCNF-graphene & $17748 \pm 6$ & $17745 \pm 7$ \\
TOCNF-graphene & $17730 \pm 6$ & $17690 \pm 6$ \\
\hline
\end{tabular}

NCNF-GO system before and after separation shows no significant difference (Table 2). Since work of adhesion is calculated by comparing energy states before and after separation, this means that the total contribution to work of adhesion from forming and breaking hydrogen bonds is close to zero. Another argument against hydrogen bond effects is that the $W_{\mathrm{A}}$ between $\mathrm{CNF}$ and graphene was quite substantial $\left(29 \mathrm{~mJ} \mathrm{~m}^{-2}\right.$, similar to dry-wetted NCNF-GO), although graphene lacks surface functionalization and cannot form any hydrogen bonds to the CNF. Thus, it is apparent that hydrogen bonding cannot be the main adhesion mechanism between NCNF and GO. This is consistent with previous simulation studies that found that hydrogen bonding is not the dominant interaction mechanism in polymer-GO interfaces. ${ }^{74,75}$

The data for TOCNF-based systems are not conclusive, since the presence of counter-ions affects hydrogen bond formation.

Let us then return to the free energy analysis based on $\Delta A^{\mathrm{ex}}$. To better understand the molecular mechanisms for strong adhesion between CNF and GO, the internal energy contribution to $W_{\mathrm{A}}$ was calculated from the difference in total potential energy before and after separation. For NCNF it was $\Delta E=-10$ $\mathrm{mJ} \mathrm{\textrm {m } ^ { - 2 }}$, and for TOCNF it was $\Delta E=-390 \mathrm{~mJ} \mathrm{~m}^{-2}$. Since these contributions are negative in both cases, these effects are working against adhesion. The largest contribution to the potential energy difference originates in the structural deformation of the CNF as it becomes adsorbed to GO, see Fig. 2 (and in-depth discussion in $\mathrm{ESI} \dagger$ ).

Since $\Delta E<0$, the dominating mechanism for the adhesion must be of entropic origin. A water molecule close to a solid attractive interface will have lower entropy than a water molecule in the liquid bulk, since its translational and rotational mobility is restricted. MD simulations of hydrated cellulose surfaces show a free energy penalty of $2-4 \mathrm{~kJ} \mathrm{~mol}^{-1}$ per water molecule. ${ }^{76,77}$ From the liquid water number density one can estimate the number of interfacial water molecules in the first hydration layer to approx. $10 \mathrm{~nm}^{-2}$, which gives a contribution of $66-133 \mathrm{~mJ} \mathrm{~m}{ }^{-2}$. In reality it is higher since the water density close to a hydrophilic surface typically is larger than in the pure liquid. ${ }^{76,77}$ This conservative estimate is large enough to dominate $W_{\mathrm{A}}$ for the case of NCNF-GO, but it becomes too small to explain adhesion for TOCNF-GO alone.

We propose that the adhesion of CNF to GO in the wet state is dominated by a gain in free energy from the reduction of interfacial water molecules upon CNF adsorption, which thus explains why a partially hydrated interface becomes weaker (Fig. 2 and Table 1). 
Such water-mediated entropic force is akin to the driving force of hydrophobic assembly. The free energy of the system $\Delta A^{\text {ex }}$ is decreased if water is removed from the interface, even for hydrophilic substrates such as cellulose. This was previously shown to be the dominating contribution to adhesion of hydrophilic xyloglucan to cellulose. ${ }^{76}$

\section{Interfacial shear strength}

Interfacial shear strength between reinforcement and surrounding matrix is a key property for composites and is critical for reinforcement pull-out mechanisms during failure, which influence tensile strength and fracture toughness. ${ }^{\mathbf{6}}$ Interfacial shear strength between the CNF and the GO was calculated from the minimum force required to initiate sliding of $\mathrm{CNF}$ on the GO in longitudinal direction, $\tau_{\max }=F_{\min } / A$, where $A$ is the contact area. Thus, $F_{\min }$ is the static friction force. A typical value for interfacial shear strength between plant fibers and polymeric matrices from micromechanics tests is 2$13 \mathrm{MPa}{ }^{78-82}$ Interfacial shear strength values for GO-GO interface is investigated theoretically in the literature ${ }^{38,83}$ and is reported to be 17-132 MPa depending on the surface chemistry of GO and the relative humidity of the environment. Just as experimental values can depend strongly on the loading rate, the $\tau_{\max }$ calculated from MD simulations can depend on the simulation time. Assuming that plastic shear deformation is an activated process, the value obtained from MD simulations represents an upper limit. For activated rate processes, slippage would occur at a lower shear force if the material system is loaded during longer time.
The simulated interfacial shear strength $\tau_{\max }$ at onset of sliding is higher for NCNF-GO than for TOCNF-GO (55.3 MPa and 41.5 MPa respectively, Table 1). Fig. $4 \mathrm{a}$ and b shows profiles of the sliding distance as a function of time for NCNF-GO and TOCNF-GO. Note that the applied force is constant and higher for NCNF-GO in Fig. 4b, since the interfacial strength is higher. The NCNF-GO nanocomposite shows so-called stick-slip behaviour during sliding, ${ }^{\mathbf{8 4}}$ which is related to breakage and formation of intermolecular bonds under shear forces (Fig. 4a). This effect is very weak for TOCNF-GO, since there is more water at this interface which facilitates sliding by lowering interfacial friction (Fig. 4b). It is not easy to quantify the amount of water at interface, since we deal with complex interfaces. However, it is evident from Fig. $2 \mathrm{a}$ and $\mathrm{b}$ that after $10 \mathrm{~ns}$ simulation in water, there is more water at TOCNF-GO interface than in NCNF-GO. This screening effect from water at the interface is also discussed by Zhang et al. ${ }^{85}$ In contrast to the PMF and work of adhesion simulations discussed in the previous section, the present interfacial shear simulations are non-equilibrium processes so that energy is dissipated in the sliding process.

In vacuum, the interfacial interactions are so strong that both NCNF and TOCNF deform under shear forces rather than sliding on the GO (Fig. 4c and d). This indicates that the CNFGO interaction energy is higher than the cohesive energy of the CNF itself, and no shear strength can be estimated for this case. Even after wetting of the dry structures in Fig. 4c and d, this deformation behaviour of cellulose nanofibers is repeated for both NCNF and TOCNF (Fig. 4e and f). The reason is that the

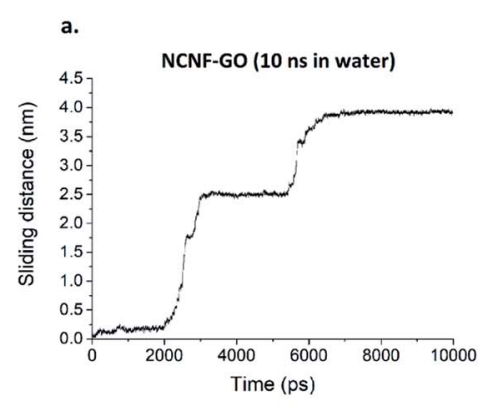

NCNF-GO

c.

Vacuum
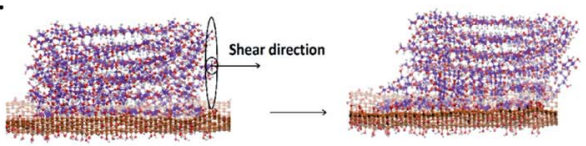

e.

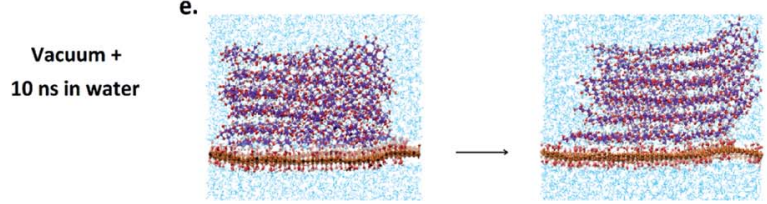

b.

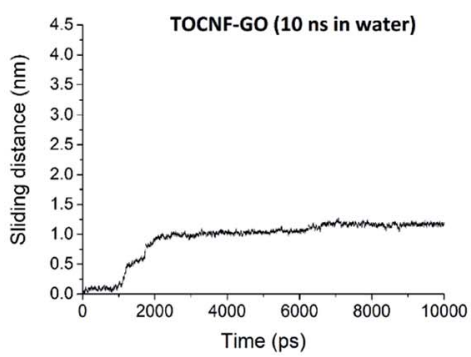

TOCNF-GO

d.

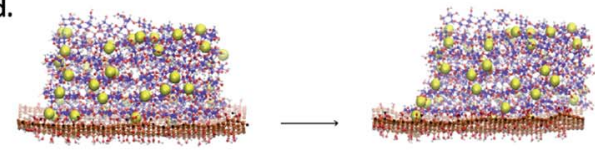

f.
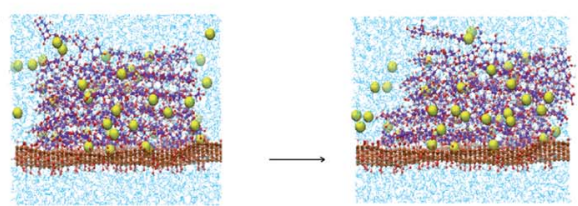

Fig. 4 Sliding profiles, sliding distance versus time, for NCNF-GO and TOCNF-GO after 10 ns in water (a and b). NCNF-GO and TOCNF-GO under shear forces in vacuum ( $c$ and $d$ ) and after vacuum $+10 \mathrm{~ns}$ in water (e and f). The shear force is applied to the centre of mass of CNF end groups. Note that the shear forces applied are different for NCNF-GO and TOCNF-GO due to differences in $\tau_{\text {max }}$. 
tight, strong interface formed between CNF and GO stays mostly intact and only a few water molecules can penetrate into the interface. This shows that the lubricating effect of water is necessary for the CNF to slide on the GO, similar to what has been reported for CNF-CNF interfaces. ${ }^{37,85,86}$

One interesting observation is that $\mathrm{CNF}$-graphene interfaces show one order of magnitude lower shear strength than CNF-GO, although they show similar work of adhesion $W_{\mathrm{A}}$, see Table 1. Both these graphene nanocomposites show very low values for interfacial shear strength: NCNF-graphene (4.8 MPa), TOCNFgraphene (3.7 MPa). One reason is the lack of hydrogen-bonding across these interfaces. Hydrogen bonds are highly directional and the $\mathrm{OH}$ groups are relatively free to change their orientation. This means they can have a significant in-plane component in contrast to dispersion forces. They can moreover break and reform on sub-nanosecond timescales. The lack of directional hydrogen bonds thus results in much lower static friction between CNF and graphene and lower $\tau_{\max }$. One may note that Zhang et $a{ }^{87}$ also reported that higher work of adhesion at the interface does not necessarily lead to higher static friction between two surfaces and the present results for the role of water clarify why this can be the case. Other contributions than hydrogen bonds to increased energy barriers for shear sliding may include increased nano-scale surface roughness. ${ }^{87,88}$

\section{Moisture effects in real CNF-GO nanocomposites}

In real nanocomposites, one would expect moisture to be trapped at the CNF-GO interface after drying. For instance, vacuum-assisted filtration, which is widely used for making CNF-GO nanopaper, ${ }^{29,34,36,42,89}$ is a relatively fast production method with insufficient time for complete drying. If real material interfaces showed as high microscale interfacial strength properties as we observed for molecular adhesion in the present study, stress-strain curves and fracture surfaces would possibly show more brittle behaviour than observed in experiments. $^{29}$ This suggests that real interfaces are likely contaminated by trapped moisture, which decreases the total work of adhesion, in line with what has been shown in the present study. It has also been suggested that moisture sorption can decrease stress transfer efficiency between cellulose nanocrystals (CNC) and graphene. ${ }^{90}$

Yet, another possible effect from water in experiments is that the ductility of the material is increased..$^{37,38,86}$ Global mechanical properties of $\mathrm{CNF}-\mathrm{GO}$ or $\mathrm{CNF} /$ graphene nanocomposites are highly sensitive to the use of NCNF or oxidized TOCNF and GO or graphene, although the present study shows that the specific nanocellulose or GO/graphene used does not strongly influence molecular adhesion properties at the interface.

There are many other 2D materials having largely hydrophilic interfaces, including layered silicates, MXenes, etc., which are used as the reinforcing phase in polymer nanocomposites. Due to advantages of water-based processing these are often combined with hydrophilic or even water-soluble polymers such as poly (vinyl alcohol), a variety of polyelectrolytes or polysaccharides including cellulose derivatives. The results presented here show that both adhesion and shear strength in such systems are likely to be highly influenced by the presence and location of residual moisture. This suggests that factors such as dispersion state, drying methods and defects dominate macroscale mechanical behaviour of this class of 2D nanocomposites.

\section{Conclusions}

Nanocomposites based on CNF nanocellulose matrix and 2D GO nanoplatelets have very large CNF/GO interface area. Since mechanical properties from experiments are sensitive to moisture, molecular adhesion effects are investigated for the $\mathrm{CNF} /$ GO and CNF/graphene interfaces, using two different CNFs. Somewhat surprising, the work of adhesion $W_{\mathrm{A}}$ is similar for all 4 combinations when the interface is dry. Water molecules at the interface gives a strong reduction in $W_{\mathrm{A}}$, with water acting as a contaminant affecting the molecular adhesion. Water can be kinetically trapped from the initial water-soaked condition, and therefore there is an effect of drying history on $W_{\mathrm{A}}$. Interestingly, hydrogen bonding has no effect on the $W_{\mathrm{A}}$. In fact, the energetic (enthalpic) balance is working against adhesion where the largest contribution originates in elastic deformation of the CNF. Instead, the $W_{\mathrm{A}}$ is controlled by the entropy gain in the material system when water molecules are released from entrapment in the interface region.

The interfacial shear strength $\tau_{\max }$ is also controlled by the presence of moisture, and failure is by a stick-slip mechanism. For dry interfaces, the strong molecular adhesion results in excessively large CNF deformation without interface failure. In contrast to the $W_{\mathrm{A}}$ simulations in dry state, hydrogen bonding is of critical importance in shear simulations due to their favourable directional nature and their ability to break and reform on short timescales.

Mechanical properties from experiments on CNF nanocellulose reinforced by GO or graphene, are highly sensitive to the specific CNF and GO reinforcement used. In contrast, the present investigation shows no effect on $W_{\mathrm{A}}$ from the type of nanocellulose or GO or graphene. This suggests that factors such as dispersion state, drying methods (entrapped water at interface) and imperfect interface contact may dominate macroscale mechanical behaviour in CNF-GO and similar 2D nanomaterials. Improved preparation methods for nanocomposites should therefore be investigated further. We also note that "real" nanocomposites will have less water close to the interface than in the present simulations. An important aspect of this investigation though, using soaked conditions, is that the critical importance of interfacial water and related mechanisms has been clarified.

\section{Conflicts of interest}

There are no conflicts to declare.

\section{Acknowledgements}

The authors acknowledge funding from the Knut and Alice Wallenberg Foundation (KAW) through the Wallenberg Wood Science Center. Computational resources were provided by the Swedish National Infrastructure for Computing (SNIC) at the 
PDC Center for High Performance Computing, KTH, partially funded by the Swedish Research Council through grant agreement no. 2016-07213.

\section{References}

1 M. Ahmadi, O. Zabihi, S. Jeon, M. Yoonessi, A. Dasari, S. Ramakrishna and M. Naebe, J. Mater. Chem. A, 2020, 8, 845-883.

2 S. Stankovich, D. A. Dikin, G. H. Dommett, K. M. Kohlhaas, E. J. Zimney, E. A. Stach, R. D. Piner, S. T. Nguyen and R. S. Ruoff, Nature, 2006, 442, 282-286.

3 M. A. Rafiee, J. Rafiee, Z. Wang, H. Song, Z.-Z. Yu and N. Koratkar, ACS Nano, 2009, 3, 3884-3890.

4 J. W. Suk, R. D. Piner, J. An and R. S. Ruoff, ACS Nano, 2010, 4, 6557-6564.

5 D. Klemm, F. Kramer, S. Moritz, T. Lindström, M. Ankerfors, D. Gray and A. Dorris, Angew. Chem., Int. Ed., 2011, 50, 54385466.

6 X. Wang, W. Xing, X. Feng, L. Song and Y. Hu, Polym. Rev., 2017, 57, 440-466.

7 W. L. Song, P. Wang, L. Cao, A. Anderson, M. J. Meziani, A. J. Farr and Y. P. Sun, Angew. Chem., Int. Ed., 2012, 51, 6498-6501.

$8 \mathrm{~K}$. Kendall, Molecular adhesion and its applications: the sticky universe, Springer Science \& Business Media, 2007.

9 J. George and H. Ishida, Prog. Polym. Sci., 2018, 86, 1-39.

10 R. O. Ritchie, Nat. Mater., 2011, 10, 817-822.

11 K. Li, C. M. Clarkson, L. Wang, Y. Liu, M. Lamm, Z. Pang, Y. Zhou, J. Qian, M. Tajvidi and D. Gardner, ACS Nano, 2021, 15, 3646-3673.

12 X. Yang, E. Jungstedt, M. S. Reid and L. A. Berglund, Macromolecules, 2021, 54, 4443-4452.

13 Z. Zhou, Q. Li and X. Zhao, Langmuir, 2006, 22, 3692-3697.

14 Y. Ogawa, Y. Nishiyama and K. Mazeau, Cellulose, 2020, 27, 9779-9786.

15 H. Sehaqui, A. Liu, Q. Zhou and L. A. Berglund, Biomacromolecules, 2010, 11, 2195-2198.

16 A. Liu, A. Walther, O. Ikkala, L. Belova and L. A. Berglund, Biomacromolecules, 2011, 12, 633-641.

17 C. Gómez-Navarro, M. Burghard and K. Kern, Nano Lett., 2008, 8, 2045-2049.

18 D. R. Dreyer, S. Park, C. W. Bielawski and R. S. Ruoff, Chem. Soc. Rev., 2010, 39, 228-240.

19 S. H. Dave, C. Gong, A. W. Robertson, J. H. Warner and J. C. Grossman, ACS Nano, 2016, 10, 7515-7522.

20 L. A. Berglund and T. Peijs, MRS Bull., 2010, 201-207.

21 R. J. Moon, A. Martini, J. Nairn, J. Simonsen and J. Youngblood, Chem. Soc. Rev., 2011, 40, 3941-3994.

22 A. Walther, F. Lossada, T. Benselfelt, K. Kriechbaum, L. Berglund, O. Ikkala, T. Saito, L. Wågberg and L. Bergström, Biomacromolecules, 2020, 21, 2536-2540.

23 A. Benítez and A. Walther, J. Mater. Chem. A, 2017, 5, 1600316024.

24 X. Gao, P. Ju, X. Liu, L. Chen, L. Ji, H. Li, H. Zhou and J. Chen, Ind. Eng. Chem. Res., 2019, 58, 5464-5471.
25 L. Zhang, Y. Zhang, F. Li, S. Yan, Z. Wang, L. Fan, G. Zhang and H. Li, ACS Appl. Mater. Interfaces, 2019, 11, 12890-12897.

26 N. V. Medhekar, A. Ramasubramaniam, R. S. Ruoff and V. B. Shenoy, ACS Nano, 2010, 4, 2300-2306.

27 S. Liu, M. Cerruti and F. Barthelat, ACS Nano, 2020, 14, 15936-15943.

28 Y. Chen, Z. Yu, Y. Ye, Y. Zhang, G. Li and F. Jiang, ACS Nano, 2021, 15, 1869-1879.

29 H. Mianehrow, G. L. Re, F. Carosio, A. Fina, P. T. Larsson, P. Chen and L. A. Berglund, J. Mater. Chem. A, 2020, 8, 17608-17620.

30 L. N. Dang and J. Seppälä, Cellulose, 2015, 22, 1799-1812.

31 F. Ren, W. Tan, Q. Duan, Y. Jin, L. Pei, P. Ren and D. Yan, Carbohydr. Polym., 2019, 209, 310-319.

32 C. Xu, G. Wang, C. Xing, L. M. Matuana and H. Zhou, BioResources, 2015, 10, 2809-2822.

33 N. D. Luong, N. Pahimanolis, U. Hippi, J. T. Korhonen, J. Ruokolainen, L.-S. Johansson, J.-D. Nam and J. Seppälä, J. Mater. Chem., 2011, 21, 13991-13998.

34 J.-M. Malho, P. i. Laaksonen, A. Walther, O. Ikkala and M. B. Linder, Biomacromolecules, 2012, 13, 1093-1099.

35 J. Duan, S. Gong, Y. Gao, X. Xie, L. Jiang and Q. Cheng, ACS Appl. Mater. Interfaces, 2016, 8, 10545-10550.

36 W. Yang, Z. Zhao, K. Wu, R. Huang, T. Liu, H. Jiang, F. Chen and Q. Fu, J. Mater. Chem. C, 2017, 5, 3748-3756.

37 Y. Hou, Q.-F. Guan, J. Xia, Z.-C. Ling, Z. He, Z.-M. Han, H.-B. Yang, P. Gu, Y. Zhu and S.-H. Yu, ACS Nano, 2020, 15, 1310-1320.

38 R. A. Soler-Crespo, W. Gao, L. Mao, H. T. Nguyen, M. R. Roenbeck, J. T. Paci, J. Huang, S. T. Nguyen and H. D. Espinosa, ACS Nano, 2018, 12, 6089-6099.

39 R. Rahman, J. Foster and A. Haque, J. Phys. Chem. A, 2013, 117, 5344-5353.

40 Q. Mao, L. Yang, X. Geng, L. Chen, B. Sapkota, H. Zhao and H. Zhu, Adv. Mater. Interfaces, 2017, 4, 1700995.

41 R. Alqus, S. J. Eichhorn and R. A. Bryce, Biomacromolecules, 2015, 16, 1771-1783.

42 C. Zhu, S. Monti and A. P. Mathew, ACS Nano, 2018, 12, 7028-7038.

43 R. Xiong, H. S. Kim, L. Zhang, V. F. Korolovych, S. Zhang, Y. G. Yingling and V. V. Tsukruk, Angew. Chem., Int. Ed., 2018, 57, 8508-8513.

44 A. Hunt, D. A. Dikin, E. Z. Kurmaev, T. D. Boyko, P. Bazylewski, G. S. Chang and A. Moewes, Adv. Funct. Mater., 2012, 22, 3950-3957.

45 A. N. Fernandes, L. H. Thomas, C. M. Altaner, P. Callow, V. T. Forsyth, D. C. Apperley, C. J. Kennedy and M. C. Jarvis, Proc. Natl. Acad. Sci. U. S. A., 2011, 108, E1195-E1203.

46 D. J. Cosgrove, Curr. Opin. Plant Biol., 2014, 22, 122-131.

47 K. Daicho, T. Saito, S. Fujisawa and A. Isogai, ACS Appl. Nano Mater., 2018, 1, 5774-5785.

48 S. Djerbi, M. Lindskog, L. Arvestad, F. Sterky and T. T. Teeri, Planta, 2005, 221, 739-746.

49 J. L. Morgan, J. Strumillo and J. Zimmer, Nature, 2013, 493, 181-186. 
50 G. Guerriero, J. Fugelstad and V. Bulone, J. Integr. Plant Biol., 2010, 52, 161-175.

51 L. H. Thomas, V. T. Forsyth, A. Šturcová, C. J. Kennedy, R. P. May, C. M. Altaner, D. C. Apperley, T. J. Wess and M. C. Jarvis, Plant Physiol., 2013, 161, 465-476.

52 M. J. Abraham, T. Murtola, R. Schulz, S. Páll, J. C. Smith, B. Hess and E. Lindahl, SoftwareX, 2015, 1, 19-25.

53 T. Darden, D. York and L. Pedersen, J. Chem. Phys., 1993, 98, 10089-10092.

54 U. Essmann, L. Perera, M. L. Berkowitz, T. Darden, H. Lee and L. G. Pedersen, J. Chem. Phys., 1995, 103, 8577-8593.

55 B. Hess, J. Chem. Theory Comput., 2008, 4, 116-122.

56 M. Parrinello and A. Rahman, J. Appl. Phys., 1981, 52, 71827190.

57 S. Nosé, Mol. Phys., 1984, 52, 255-268.

58 W. G. Hoover, Phys. Rev. A: At., Mol., Opt. Phys., 1985, 31, 1695.

59 K. N. Kirschner, A. B. Yongye, S. M. Tschampel, J. González-Outeiriño, C. R. Daniels, B. L. Foley and R. J. Woods, J. Comput. Chem., 2008, 29, 622-655.

60 J. Wang, R. M. Wolf, J. W. Caldwell, P. A. Kollman and D. A. Case, J. Comput. Chem., 2004, 25, 1157-1174.

$61 \mathrm{~W}$. L. Jorgensen, J. Chandrasekhar, J. D. Madura, R. W. Impey and M. L. Klein, J. Chem. Phys., 1983, 79, 926935.

62 D. E. Smith and L. X. Dang, J. Chem. Phys., 1994, 100, 37573766.

63 W. Humphrey, A. Dalke and K. Schulten, J. Mol. Graphics, 1996, 14, 33-38.

64 S. Kumar, J. M. Rosenberg, D. Bouzida, R. H. Swendsen and P. A. Kollman, J. Comput. Chem., 1992, 13, 1011-1021.

65 J. S. Hub, B. L. De Groot and D. Van Der Spoel, J. Chem. Theory Comput., 2010, 6, 3713-3720.

66 A. Kelly and P. Sciences, Proc. R. Soc. London, Ser. A, 1970, 319, 95-116.

67 Z. Wei, R. Sinko, S. Keten and E. Luijten, ACS Appl. Mater. Interfaces, 2018, 10, 8349-8358.

68 M. Garg, M. Linares and I. Zozoulenko, Biomacromolecules, 2020, 21, 3069-3080.

69 C. Moser, G. Henriksson and M. Lindström, Nord. Pulp Pap. Res. J., 2018, 33, 647-650.

70 H. Nilsson, S. Galland, P. T. Larsson, E. K. Gamstedt, T. Nishino, L. A. Berglund and T. Iversen, Compos. Sci. Technol., 2010, 70, 1704-1712.
71 R. H. Newman, Cellulose, 2004, 11, 45-52.

72 P. Chen, G. L. Re, L. A. Berglund and J. Wohlert, J. Mater. Chem. A, 2020, 8, 23617-23627.

73 E. Peris, J. C. Lee, J. R. Rambo, O. Eisenstein and R. H. Crabtree, J. Am. Chem. Soc., 1995, 117, 3485-3491.

74 X. Zhang, H. Nguyen, M. Daly, S. T. Nguyen and H. D. Espinosa, Nanoscale, 2019, 11, 12305-12316.

75 J. Y. Choi, X. Zhang, H. T. Nguyen, M. R. Roenbeck, L. Mao, R. Soler-Crespo, S. T. Nguyen and H. D. Espinosa, J. Mech. Phys. Solids, 2021, 156, 104578.

76 S. Kishani, T. Benselfelt, L. Wågberg and J. Wohlert, J. Colloid Interface Sci., 2020, 588, 485-493.

77 S.-T. Lin, M. Blanco and W. A. Goddard III, J. Chem. Phys., 2003, 119, 11792-11805.

78 R. B. Adusumali, M. Reifferscheid, H. Weber, T. Roeder, H. Sixta and W. Gindl, 2006.

79 H. A. Khalil, H. Ismail, H. Rozman and M. Ahmad, Eur. Polym. J., 2001, 37, 1037-1045.

80 D. Cho, S. B. Yoon and T. Drzal, Compos. Interfaces, 2009, 16, 769-779.

81 J. Trejo-O'Reilly, J. Cavaille, M. Paillet, A. Gandini, P. Herrera-Franco and J. Cauich, Polym. Compos., 2000, 21, 65-71.

82 J. Karlsson, P. Gatenholm, J. F. Blachot and A. Peguy, Polym. Compos., 1996, 17, 300-304.

83 M. Daly, C. Cao, H. Sun, Y. Sun, T. Filleter and C. V. Singh, ACS Nano, 2016, 10, 1939-1947.

84 J. Keckes, I. Burgert, K. Frühmann, M. Müller, K. Kölln, M. Hamilton, M. Burghammer, S. V. Roth, S. StanzlTschegg and P. Fratzl, Nat. Mater., 2003, 2, 810-813.

85 C. Zhang, S. Keten, D. Derome and J. Carmeliet, Carbohydr. Polym., 2021, 117682.

86 R. Sinko and S. Keten, Appl. Phys. Lett., 2014, 105, 243702.

87 Q. Zhang, Y. Qi, L. G. Hector Jr, T. Cagin and W. A. Goddard

III, Phys. Rev. B: Condens. Matter Mater. Phys., 2007, 75, 144114.

88 I.-S. Byun, D. Yoon, J. S. Choi, I. Hwang, D. H. Lee, M. J. Lee, T. Kawai, Y.-W. Son, Q. Jia and H. Cheong, ACS Nano, 2011, 5, 6417-6424.

89 P. Laaksonen, A. Walther, J. M. Malho, M. Kainlauri, O. Ikkala and M. B. Linder, Angew. Chem., Int. Ed., 2011, 50, 8688-8691.

90 J. Morgan, M. Craciun and S. Eichhorn, Compos. Sci. Technol., 2019, 177, 34-40. 